\title{
Evaluation d'un dispositif expérimental d'évaluation certificative des étudiants en fin de troisième cycle de médecine générale
}

\author{
Evaluation of an Experimental Assessment System for the Certification of Students in General \\ Medicine at the end of the Postgraduate Curriculum
}

Pierre LE MAUFF',2, Pierre POTTIER ${ }^{1,2}$, Lionel GORONFLOT' ${ }^{1}$, Jacques BARRIER ${ }^{2}$

\begin{abstract}
Résumé Contexte : Le nouveau dispositif pédagogique du troisième cycle de médecine générale à la faculté de médecine de Nantes est désormais davantage centré sur les apprentissages. Une promotion de résidents a pu choisir soit les anciennes soit les nouvelles modalités d'évaluation. Sujets et méthodes : Nous avons réalisé trois groupes de discussion avec des résidents qui ont passé l'épreuve, avec les enseignants qui les ont évalués et avec les responsables facultaires. Résultats : Tous les groupes ont apprécié les nouvelles modalités comme étant moins scolaires, plus impliquantes, plus centrées sur le parcours personnel et sur la pratique professionnelle, renseignant plus largement sur l'acquisition des compétences. Les internes ont trouvé difficile l'auto-évaluation, l'auto-formation et la constitution d'un portfolio de bonne qualité ; ils ont souligné la nécessité de l'aide d'un tuteur pédagogique. Les enseignants ont souligné la nécessité d'une cohérence globale du dispositif; le portfolio doit être correctement structuré et documenté progressivement tout au long du cursus. Les responsables facultaires ont souligné la nécessaire mobilisation de tous les maîtres de stage, généralistes et hospitalier. Conclusion : En dépit de plusieurs faiblesses méthodologiques, ce travail a permis d'établir la viabilité opérationnelle du nouveau dispositif, son acceptabilité par les étudiants et par les formateurs concernés, sa compréhension par les responsables institutionnels. Plusieurs pistes d'amélioration du dispositif ont été identifiées.
\end{abstract}

Mots-clés Evaluation ; certification ; résidents ; médecine générale ; portfolio.

Abstract Context: The pedagogical method used in post-graduate curriculum of general medicine at the Faculty of medicine of Nantes have changed toward student-centered active-based learning. Somme residents could validate their competencies in accordance to old or new methods. Methods: We have formed three focus groups with respectively residents who passed the test, teachers who have assessed these residents and Faculty officials. Results: All groups reported a positive appreciation of the new pedagogical approach. All groups were in agreement on several points: it is less tutorial, it raises more involvement, it requests the focus of personal path and professional practice and it gives reliable information about competency acquisition. Interns have experienced some difficulties to evaluate themselves, to take the responsibility of their own formation and to develop a suitable portfolio. They have pointed out the need for pedagogical support. Medical teachers have emphasized the need for a global coherence of the new pedagogical method: they have reported the importance of an appropriate portfolio structure and documentation, in accordance with the progression of competencies along the formation. Faculty officials underlined the need for the mobilization of tutors coming from different medical practice environments (hospital as well as general medicine). Conclusion: Even with methodological weaknesses, this work has demonstrated the practicability of a new pedagogical approach. It was accepted by third year residents and general practice trainers involved with their assessment. The novel pedagogical approach was also considered by Faculty officials. Several leads for upcoming improvements have been identified.

Key words Evaluation; certification; residents; general medicine; portfolio.

Pédagogie Médicale 2006;7:142-54

1- Département de médecine générale, Faculté de médecine - 44000 Nantes - France

2- Unité de développement et de recherche pédagogique, même adresse

Correspondance : Pierre Le Mauff - Département de médecine générale, Faculté de médecine de Nantes - 44000 Nantes - France.

Téléphone : 0240412826 - Mailto:plemauff@wanadoo.fr 


\section{Contexte et problématique pédagogique}

Les résidents qui ont terminé leur troisième cycle de médecine générale (formation post-graduée) à la faculté de médecine de l'Université de Nantes (France) en 20032004 ont eu la possibilité de valider leur cursus selon deux modalités, au choix :

- s'ils choisissaient de se conformer aux exigences de l'ancien dispositif de validation, les étudiants devaient : 1) valider l'ensemble des stages prévus par la maquette de troisième cycle (quatre semestres hospitaliers dont un en médecine interne ou gériatrie, un en médecine d'urgence, un en pôle mère-enfant, un semestre libre et deux semestres en médecine générale ambulatoire) ;2) réaliser un travail personnel à type de mémoire pendant le semestre de stage obligatoire en médecine générale ; 3) participer au moins à deux tiers des séances d'enseignement théorique (participation attestée par la signature des enseignants sur un carnet de présence). Si un résident ne pouvait justifier d'avoir assisté au quota minimum d'enseignements, il était soumis à un examen clinique objectif structuré (ECOS) constitué d'une douzaine de stations.

- s'ils choisissaient de se conformer aux exigences du dispositif expérimental de validation, ils devaient : 1) valider les stages prévus par la maquette réglementaire du troisième cycle de médecine générale et réaliser travail personnel à type de mémoire pendant le stage chez le praticien - comme précédemment - ;3) effectuer une présentation orale devant un jury pluridisciplinaire composé de quatre maîtres d'apprentissages (deux médecins hospitaliers et deux médecins de soins primaires ambulatoires). Le résident commentait son cursus de troisième cycle, ses choix de lieux de stage, les points forts et les points faibles de sa formation, ses travaux de recherche personnels. Le jury analysait le document d'auto-évaluation que l'interne devait préalablement élaborer à partir d'un guide et le contenu de son portfolio. Une vignette clinique, exemplaire d'une situation complexe de médecine générale, était ensuite proposée à l'interne ; il lui était demandé d'expliciter et d'argumenter la stratégie de prise en charge qu'il préconisait. Le jury prononçait la validation du cursus en s'appuyant sur un jugement global prenant en compte : les traces d'apprentissages rapportées dans son portfolio, les avis de validation des stages formulés par les maîtres d'apprentissages, une appréciation de sa capacité de synthèse et de prise de décision par rapport à la situation clinique faisant l'objet de la vignette proposée. En cas de non-validation, l'interne représentait son dossier plus tard, avec la consigne de le compléter en tenant compte des commentaires et des recommandations du jury. Le dispositif expérimental répondait au souhait que les modalités d'évaluation certificative des étudiants soient davantage congruentes avec les orientations pédagogiques du département de médecine générale de la faculté. En cohérence avec l'intention de former des praticiens réflexifs, le curriculum du troisième cycle a en effet été réorganisé en s'appuyant respectivement sur une approche constructiviste de l'apprentissage et sur la perspective de l'enseignement contextualisé ${ }^{1}$. Ce choix a induit des transformations des modalités pédagogiques de l'enseignement, de l'évaluation et de la validation des apprentissages $^{2,3}$. La planification de cette (r)évolution étant faite, il nous est apparu indispensable de la mettre en place de façon expérimentale dès l'année universitaire 2003-2004. Les résidents de médecine générale " ancien régime " ont eu dès lors le choix de valider leur troisième cycle de médecine générale selon les modalités habituelles ou selon les nouvelles modalités.

Lobjectif du présent travail est d'évaluer cette expérimentation pour identifier les difficultés rencontrées, les problèmes à résoudre, les améliorations à apporter, à partir d'un recueil des perceptions des résidents et des enseignants qui ont participé au dispositif, dans une démarchequalité d'amélioration des pratiques.

\section{Sujets et méthodes}

Des entretiens répondant aux principes et au format des groupes de discussion ${ }^{4,5}$ (focus group) ont été effectués respectivement auprès de trois groupes : des résidents de médecine générale qui avaient choisi le dispositif expérimental d'évaluation, les enseignants qui avaient participé au jury et les responsables de la faculté de médecine. Le groupe des résidents était constitué des dix internes qui venaient de passer l'épreuve.

Tous ont accepté de participer à cet entretien de groupe et il a eu lieu le jour même de l'épreuve, dans les locaux de la faculté. Le groupe des enseignants était constitué de huit des douze enseignants (généralistes et hospitaliers) qui ont constitué les trois jurys. Il a eu lieu un mois après l'épreuve, dans les locaux de la faculté. Le groupe des responsables de la faculté était constitué de six personnes : le doyen de la faculté, le vice-doyen chargé des études, le directeur du département de formation médicale continue et de pédagogie médicale, le directeur de l'unité de recherche et de développement pédagogique, le directeur du département de médecine générale, le coordinateur universitaire du département de médecine générale. Pour des raisons de disponibilité des membres pressentis, l'entretien de groupe n'a pu se dérouler que trois mois après l'épreuve. 


\section{Recherche et Perspectives}

Les groupes de discussion étaient animés par un modérateur et leur durée était d'environ une heure. Un observateur notait les éléments de communication non verbale. Le guide d'entretien avait été validé auprès d'enseignants du département de médecine générale et du département de formation médicale continue et de pédagogie médicale trois semaines avant l'épreuve. Les trois enregistrements ont été intégralement transcrits et chaque ligne numérotée pour permettre de bien référencer le verbatim de chaque groupe de discussion. Nous avons constitué une grille d'analyse selon les principes décrits par Giami ${ }^{6}$, en quatre étapes : 1) établissement d'une liste structurée de catégories d'analyse permettant de découper le verbation de l'entretien en séquences explorant le même champ d'analyse ; 2) découpage des catégories d'analyse en unités d'analyse. Habituellement il se fait selon deux modalités : soit selon une logique formelle et il prend en compte des mots clés ou des propositions grammaticales, soit selon une logique de significativité et il prend en compte des thèmes. C'est cette deuxième approche que nous avons choisi, en considérant le thème comme "unité minimale de signification ". Cette approche nous a paru plus opératoire, permettant de regrouper sans trop de difficultés des passages de sens identique qu'il aurait été beaucoup plus difficile d'identifier à partir de mots ou de groupes de mots, les différents participants ne partageant pas le même lexique ou le même vocabulaire ; 3) articulation de la problématique et des données recueillies, ce qui permet de ne pas rester prisonnier de la logique propre de l'entretien. L'objectif était de regrouper les unités minimales de signification de même sens dispersées dans l'analyse chronologique de l'entretien ; 4) intégration des données échappant à la classification. Les étapes précédentes de classement et d'analyse ont laissé de côté du matériel informatif (silences, murmures, onomatopées, locutions brèves -oui, $\mathrm{OK}$, non, d'accord, peut-être,...-) que nous avons réintégré dans la mesure où il avait du sens, venant renforcer ou modérer le discours des participants. Enfin les éléments de la communication non verbale, enregistrés par l'observateur ont été intégrés.

Le travail de découpage des processus d'énonciation puis d'articulation en unités de signification s'est fait à l'aide du logiciel Microsoft Word ${ }^{\circledR}$. Linterprétation globale, à partir de la réintroduction des données non classifiées, a été effectuée à la fois de façon libre et à l'aide du logiciel Microsoft Word ${ }^{\circledR}$.

\section{Résultats}

\section{Perceptions et propositions des résidents}

Même si les résidents ont manifesté leur lassitude d'être évalués une fois de plus, ils ont vécu cette épreuve de validation de façon positive, considérant que ces modalités étaient intéressantes, les impliquant davantage que les précédentes. Ce changement a parfois été perçu comme étant radical, au point que certains se sont demandés s'il s'agissait bien d'une évaluation tandis que d'autres ont trouvé dommage que cela n'ait pas eu lieu plus tôt dans leur troisième cycle. L'observateur a noté que les différents acteurs se sont largement exprimés pendant cette séquence, respectant bien la prise de parole des uns et des autres. La seule période un peu " animée " est celle où un participant s'est interrogé sur le fait de savoir si ces modalités constituaient vraiment une évaluation : le groupe s'est scindé en deux fractions inégales, la majorité des résidents acceptant sans réserve cette procédure, une minorité émettant des réserves.

Les résidents ont plébiscité le processus d'auto-évaluation, même s'ils l'ont trouvé difficile, en notant qu'il autorise potentiellement la falsification. Ils ont demandé l'assistance d'un enseignant (tuteur) pour mener à bien une évaluation précoce et continue. Elle devrait se faire en situation professionnelle, pendant les stages, avec une rétroaction (feedback) des maîtres de stage. Pour les résidents, les modalités de l'évaluation doivent être clairement définies dès le départ. Elles sont incitatives et de nature à modifier leur comportement. Selon eux, la validation devrait être finale et les tuteurs qui assurent l'évaluation formative pendant le troisième cycle ne peuvent être ceux qui prononcent la validation certificative finale. Il s'est avéré que les internes connaissaient mal les différents outils employés dans le dispositif : les ECOS, le portfolio, le guide d'auto-évaluation. Les ECOS leur paraissent un moyen objectif d'évaluer les compétences cliniques. Le principe du portfolio leur paraissait impliquant et responsabilisant. Cependant, en pratique, ils n'avaient pas une connaissance claire des consignes de son utilisation, ne connaissant pas avec précision la nature du matériau à collecter, par exemple. Ils réclamaient donc une information et une structuration de celui-ci. Le guide d'auto-évaluation était apprécié même si certains items leur paraissaient à modifier. L'observateur a noté pendant cette séquence l'émergence de deux leaders et la mise en retrait de trois résidents qui ne s'exprimaient que sur sollicitation du modérateur et pauvrement. Il y a eu plusieurs périodes de verbalisations difficiles, entrecoupées de silences témoignant d'un manque de connaissances sur le concept d'évaluation, sur les outils d'évaluation et en particulier sur le portfolio.

Les résidents ont demandé la suppression de l'épreuve 
organisée autour de la vignette clinique, en souhaitant que l'évaluation des compétences cliniques soit faite en amont, en situation professionnelle pendant les stages. Selon eux, l'épreuve finale doit être un entretien avec un jury qui apprécie le parcours et évalue le contenu du portfolio. La décision doit être commentée par le jury. L'observateur a noté un démarrage difficile de la discussion, la prise de parole des deux leaders identifiés précédemment permettant aux autres de se positionner, soit en validant les propos (le plus souvent), soit en exprimant leur absence d'avis, soit en restant silencieux.

\section{Perceptions et propositions des enseignants}

Les enseignants ont souligné que l'ambiance de l'épreuve avait été conditionnée par le positionnement initial du jury, allant de la décontraction à la solennité, celle-ci étant source de malaise pour certains enseignants. Le dispositif expérimental a été considéré comme apportant un progrès par rapport au dispositif antérieur car le champ d'exploration a été plus large. L'implication personnelle de l'apprenant a été plus importante ; il a été davantage considéré comme un professionnel en formation (autonomisation). Selon les enseignants, un tel dispositif permet une évaluation sur la durée. L'insuffisance des traces d'apprentissages apportées par les résidents a été mise en exergue. Une des explications avancées par les enseignants concerne le fait que le changement des modalités d'évaluation est survenu en cours de troisième cycle, ne laissant pas le temps aux étudiants de constituer un portfolio suffisamment documenté. La faisabilité de l'épreuve a été jugée bonne ; sa " fiabilité » est conditionnée par la qualité du contenu du portfolio. Les enseignants ont souligné qu'une seule vignette clinique était insuffisante pour évaluer avec rigueur les compétences cliniques des étudiants mais ils ont remarqué que les étudiants qui argumentaient leur curriculum et qui faisaient état d'un projet clair en médecine générale étaient les plus performants sur l'épreuve de vignette clinique. A l'inverse, les étudiants qui n'ont pas été performants sur la vignette clinique avaient eu des difficultés à argumenter leur parcours lors de l'entretien et ne semblaient pas être motivés par l'exercice de la médecine générale. L'observateur a noté que le groupe a fonctionné d'emblée, sans temps de latence, chaque participant s'exprimant librement, avec une écoute de grande qualité de la part de tous les participants.

Les enseignants pensaient que seule l'évaluation réalisée en situation professionnelle permettait d'évaluer de façon pertinente les compétences cliniques des étudiants. Selon eux, l'évaluation devrait être prédictive, c'est-à-dire déterminer si le résident fera un bon professionnel. Pour cela, il conviendrait d'évaluer sa capacité à raisonner et sa capacité à évaluer sa pratique professionnelle (avec l'aide du tuteur). Les enseignants pensaient que cela passerait par la structuration du portfolio, au moyen de consignes concernant les travaux et tâches personnels à documenter par les apprenants. A cet égard, ils ont souligné l'intérêt qu'ils rapportent des récits de situations complexes et authentique $^{8}$ (Annexe 1). Le groupe des enseignants a posé la question suivante : quel est le poids d'une évaluation finale face à une évaluation continue sur trois ans ? Les enseignants ont distingué l'évaluation formative de la validation (certification). Ils ont défini la certification comme une évaluation sommative sanctionnelle finale à partir de plusieurs éléments : auto-évaluation de l'interne, évaluations par tous les enseignants (maîtres de stage, tuteur), prise en compte de tâches et de travaux personnels. L'intérêt d'une évaluation intermédiaire par un jury a été évoqué. Elle aurait vocation à être organisée en cas de problème, à la demande du résident et/ou du tuteur. Selon les enseignants, le portfolio devrait contenir les résultats des évaluations des compétences faites pendant les stages, des évaluations formatives du tuteur, les travaux personnels de l'étudiant, les tâches prescrites par les différents maîtres de stage ou par le tuteur. D'autres éléments pourraient y figurer : les évaluations critériées de ses stages par l'interne - nous utilisons la grille de Bordage ${ }^{9}$ pour les stages chez le praticien (Annexe 2) et nous mettons au point une grille d'évaluation des stages hospitaliers selon la même méthode avec le département de pédagogie -, des appréciations rendant compte du point de vue des patients et du médecin remplacé en cas de remplacement.

Sous réserve que les compétences cliniques aient été évaluées pendant les différents stages, les enseignants recommandent la suppression de l'épreuve de vignette clinique. L'analyse du portfolio pourrait être faite avant la séance de présentation orale devant le jury par l'un de ses membres qui en ferait le rapport le jour de l'épreuve. La qualité du portfolio pourrait être optimisée en y documentant de façon explicite les résultats des interventions et des rétroactions récurrentes des divers maitres d'apprentissages et des tuteurs. Le jury devrait restituer à l'étudiant des commentaires pour l'aider à se situer par rapport aux contraintes professionnelles et souligner les points à renforcer pour devenir un bon professionnel, ce qui permet de mettre la formation médicale continue en perspective. L'observateur a noté que le groupe a fonctionné de façon très respectueuse, chacun des intervenants pouvant s'exprimer très librement et développer son argumentaire. Aucun leader ne s'est dégagé.

\section{Perceptions et propositions des responsables facultaires \\ Les responsables facultaires ont bien perçu le caractère}


expérimental - au sens où il s'agit d'un projet en cours de développement - et donc du caractère évolutif de cette épreuve de validation. Ils nous ont remercié de faire ce travail " qui va dans le bon sens », les modalités antérieures étant jugées archaïques. Ils se sont sentis impliqués dans cette démarche de recherche et développement, en participant à ce travail d'analyse critique et en apportant leur expertise. Ils ont reconnu enfin, qu'en l'état et en dépit de ses imperfections, cette épreuve était un progrès par rapport aux anciennes modalités. L'observateur a noté l'absence d'interaction entre les prises de parole, chaque intervenant prenant la parole à tour de rôle et exposant ses commentaires de manière " académique ".

Les finalités de l'évaluation ont été rappelées : permettre de guider les apprentissages des apprenants (finalité formative) et de porter un jugement sur leurs compétences professionnelles (finalité à la fois formative et sommative - sanctionnante -), avec l'objectif d'attester la compétences professionnelle des diplômés. Ils y ont ajouté un objectif institutionnel, celui de permettre un repérage des internes susceptibles de devenir à leur tour enseignants de la discipline. Les responsables ont souligné la nécessité de "bien évaluer le fond autant que d'évaluer le professionnalisme ". Les terrains de stages hospitaliers leur paraissaient sous utilisés au profit des stages en soins primaires et des enseignements décontextualisés. Ils ont rappelé que les résidents de médecine générale effectuaient au moins quatre stages sur six dans les hôpitaux et qu'il fallait profiter de ces mises en situations professionnelles (évaluation authentique). Les outils d'évaluation ont ensuite été analysés. Pendant les stages, différentes traces d'apprentissages peuvent être appréciées : les récits de situations cliniques et professionnelles, les participations aux groupes d'échanges de pratiques, les travaux personnels. Une réflexion a également eu lieu sur les moyens humains, les maîtres de stage et les tuteurs, et sur l'outil de recueil de toutes ces traces d'apprentissages, le portfolio. La validation des compétences cliniques devrait être faite avant l'épreuve de présentation orale devant le jury. En cas de démonstration insuffisante de telles compétences, se poserait la question d'organiser à des épreuves complémentaires de type ECOS. Selon les responsables facultaires, cette alternative devrait être exceptionnelle si des évaluations récurrentes adéquates, impliquant maîtres de stage et tuteur, sont préalablement organisées. L'analyse du cursus semble pouvoir être informative mais peut être redondante avec les évaluations des maîtres de stage. L'analyse du portfolio est perçue comme devant être l'élément clé de la validation. L'observateur a noté que le fonctionnement du groupe s'est graduellement libéré, un débat s'établissant entre les différents participants. Deux leaders ont émergé : l'un (le doyen) à propos des enjeux institutionnels et politiques et l'autre (le directeur de l'unité de recherche et de développement pédagogique) à propos des enjeux pédagogiques.

Les responsables considèrent que les modalités doivent être allégées et simplifiées. Il est proposé une réduction des membres du jury de quatre à deux enseignants : un hospitalier et un généraliste. L'analyse du portfolio pourrait être faite avant la présentation devant le jury par un enseignant de médecine générale n'ayant pas participé à l'évaluation formative de l'interne, qui en ferait un rapport au jury. Les évaluations des compétences cliniques devraient avoir été faites antérieurement. Admettant que le portfolio peut avoir vocation à devenir l'élément de validation principal, les responsables considèrent qu'il conviendrait d'en améliorer la structuration et le contenu. Il est apparu nécessaire aux responsables de la faculté de favoriser une "imprégnation des praticiens hospitaliers aux concepts de la médecine générale ", par le biais d'une réflexion pédagogique menée dans les services en partenariat avec le département de médecine générale, une stimulation de la vocation enseignante de tous les maîtres de stage avec appropriation des concepts de l'évaluation formative. L'observateur a noté que le groupe a fonctionné de manière très détendue pendant cette séquence sans émergence de leader, chacun s'exprimant librement et avec humour le plus souvent.

\section{Discussion}

\section{Limites méthodologiques}

Notre travail s'inscrit dans une approche qualitative et, dans ce cadre, nous avons choisi de recueillir les informations pertinentes à l'aide d'entretiens conduits selon le format et les principes du groupe de discussion. Des alternatives méthodologiques pouvaient être discutées ; ainsi, nous aurions pu interroger un échantillon ou la totalité des personnes concernées à l'aide d'un questionnaire ou encore recourir à des entretiens individuels semi-structurés. Le choix des groupes de discussion répondait au souhait de privilégier un recueil « dynamique " des données, les interactions individuelles au sein du groupe étant exploitées pour enrichir la production de chacune des personnes constituant le groupe. Certaines limites méritent cependant d'être soulignées. A la suite de Lincoln et Guba ${ }^{10}$, il est habituel de discuter pour ce type d'études la crédibilité et la transférabilité ${ }^{11}$. 
Concernant le critère de crédibilité, un biais potentiel est constitué par l'impossibilité de conduire les trois groupes de discussion en même temps, ce qui permet potentiellement les fuites et donc la contamination éventuelle des deuxième et troisième entretiens. Ce risque nous semble minime pour ce qui concerne les interactions potentielles entre le groupe des étudiants et les deux autres (enseignants et responsables facultaires), les résidents de médecine générale n'ayant, à ce moment, que très peu de contact avec les enseignants puisqu'ils ont terminé leurs études et qu'ils sont en remplacement à cette époque de l'année. Il n'en est pas de même pour ce qui concerne le deuxième et le troisième groupes : les praticiens hospitaliers, les enseignants de médecine générale et les responsables de la faculté de médecine ayant des contacts réguliers, on ne peut exclure des échanges entre eux. D'autres limites sont plus sérieuses. L'une d'entre elles est liée à une triangulation non optimale de nos sources de données. Trois populations différentes ont certes été interrogées et les interprétations effectuées à partir des productions de chacune d'entre elles ont pu être mises en perspective au moment de l'interprétation. En revanche, les points de vue de chaque population n'ont été explorés que d'une seule manière. Par ailleurs, la validation par les répondants des interprétations élaborées à partir des verbatims n'a pu être effectuée que pour le groupe des enseignants et pour celui des responsables facultaires; en raison de la difficulté à contacter les étudiants diplômés après la fin de leurs études, elle n'a pas pu être effectuée pour le groupe des résidents. On peut également, au moins à titre de réserve de principe, s'interroger sur la neutralité et l'objectivité de l'animation des groupes de discussion, les animateurs (un professeur des universités-praticien hospitalier, un maître de conférences des universités associé-médecin généraliste) ayant été recrutés parmi des personnes impliquées dans la planification du dispositif expérimental de validation qui fait l'objet de cette évaluation. En tant que telles, ces faiblesses méthodologiques peuvent avoir altéré la crédibilité de nos résultats. Nous avions accepté délibérément cette limite dans la mesure où il était important pour nous de disposer de ces résultats suffisamment tôt pour préparer la mise en place du Diplôme d'études spécialisées de médecine générale, à partir de novembre 2004.

En revanche, la similitude de notre contexte d'étude, tel que nous l'avons rappelé, avec ceux de nombreux autres départements de médecine générale de facultés françaises est à nos yeux le gage d'un certain degré de transférabilité des résultats.

\section{Signification des résultats}

Les perceptions des trois groupes interrogés à l'égard du dispositif expérimental de validation certificative du cursus post-gradué de médecine générale mis en place sont convergentes. Elles témoignent d'une impression favorable, notamment à l'égard du fait que, par rapport au dispositif antérieur, les participants étaient plus impliqués, l'évaluation était plus centrée sur la démarche professionnelle, les modalités permettaient de renseigner plus largement sur les compétences acquises. Cette perception est en accord avec l'évolution des orientations conceptuelles décidée au sein du département de médecine générale : l'évaluation qui était jusqu'à présent centrée sur l'enseignement est maintenant centrée sur les apprentissages réalisés par les étudiants. Ce changement a été clairement perçu par certains étudiants comme une véritable rupture puisqu'ils se demandaient s'ils avaient "vraiment " participés à une évaluation.

Les trois groupes partagent et valident la vision d'une évaluation répondant davantage aux caractéristiques d'une évaluation authentique, en cohérence avec le paradigme d'apprentissage. Ces caractéristiques ont notamment été définies par Wiggins ${ }^{12}$ : procédure destinée à un jury, tâches et exigences connues avant la situation d'évaluation, collaboration avec les pairs, évaluation de tâches contextualisées, prise en considération des stratégies cognitives et métacognitives à partir de problèmes complexes, critères multiples, l'auto-évaluation faisant partie de l'évaluation. Ainsi, la description faite par le groupe des internes de l'évaluation idéale de leurs compétences met en exergue les éléments suivants : règles du jeu connues à l'avance, évaluation formative par les maîtres de stage et le tuteur, en situation professionnelle ou à partir de tâches contextualisées, auto-évaluation avec assistance du tuteur. Le groupe des enseignants souligne également ces notions d'évaluation formative et d'évaluation authentique ; il insiste en outre sur les notions d'évaluation prédictive "pour déterminer si les internes feront de bons professionnels " et d'évaluation "sommative, sanctionnante", qui appartiennent au concept de certification. Dans sa définition de l'évaluation, Gardner ${ }^{13}$ souligne cette double finalité de l'évaluation, l'une destinée aux apprenants et aux enseignants, l'autre destinée aux institutions, l'une à visée " diagnostique " pour permettre aux apprenants et aux enseignants de " réguler » respectivement leurs apprentissages et leurs enseignements, l'autre à visée " pronostique " pour déterminer si un résident a acquis les compétences nécessaires à son futur exercice 


\section{Recherche et Perspectives}

professionnel ${ }^{14}$. Le groupe des responsables facultaires enrichit la discussion sur l'évaluation par l'analyse de l'utilisation des terrains de stages, en particulier hospitaliers, où nombre de situations cliniques pourraient être exploitées au service du développement des compétences des étudiants. Cela supposerait idéalement une meilleure appropriation des concepts et des outils pédagogiques mis en place par le département de médecine générale de la part des maîtres de stage. Des objectifs de formation transversaux, spécifiquement orientés vers l'exercice de la médecine générale, devraient être définis pour chaque stage, en plus des aspects spécifiques nécessaires au soin des patients hospitalisés dans les différents services. L'importance de bien définir les composantes de l'apprentissage et l'encadrement à mettre en place a été soulignée dans la littérature ${ }^{1,15}$. Ce n'est pas la réalité d'aujourd'hui dans notre contexte institutionnel. Il reste un gros travail d'information et de formation à faire, dont les responsables facultaires ont conscience. Ils nous encouragent à " utiliser la bannière institutionnelle " pour ce faire. La réflexion de ce groupe est allée au-delà de l'évaluation des compétences cliniques pour aborder le professionnalisme ${ }^{16}$ et pour évoquer une préoccupation institutionnelle, celle du repérage des futurs enseignants de la discipline.

Concernant les outils d'évaluation des apprentissages, les internes ont des représentations très limitées, puisqu'ils ne citent que les outils qu'ils ont vu mettre en œuvre, avec un niveau de connaissance et d'appropriation faible. Les enseignants et les responsables facultaires ont souligné l'importance de l'évaluation du raisonnement de l'apprenant, avec l'intérêt des supervisions du résident en action professionnelle mais aussi de l'analyse de ses travaux et de ses tâches réflexives. Ils rejoignent en cela certaines exigences formulées par Tardif ${ }^{17}$ : "Seules les tâches complètes, complexes et signifiantes pour l'apprenant peuvent constituer une situation valide d'évaluation sur le plan cognitif ". La construction du portfolio, régulièrement accompagnée et évaluée par le tuteur, incluant les travaux personnels et les tâches d'apprentissages réalisés pendant les stages, sur toute la durée du cursus, est en cohérence avec la perspective de l'évaluation authentique. Les trois groupes ont dénoncé le manque de structuration du portfolio et le manque d'appropriation de l'outil par les résidents. Ce point s'explique sans doute en partie par le fait que ce portfolio sur support papier n'a été mis en place qu'une année auparavant, alors que les résidents avaient déjà accompli deux années de leur cursus. L'élaboration du portfolio $a$ posteriori était donc difficile; elle est même totalement antinomique avec les principes de l'outil. Nous faisons l'hypothèse que le portfolio actuel, élaboré sur support électronique, appartenant à l'interne, avec un code d'accès sécurisé, structuré en trois niveaux, devrait pallier un certain nombre de ces difficultés (Annexe 3). L'utilisation du portfolio comme outil d'évaluation certificative fait actuellement l'objet d'un débat ${ }^{18}$; en tout état de cause, il conviendra d'approfondir la réflexion sur les conditions et les exigences à mettre en place pour que cet usage soit légitime dans le cadre de notre dispositif certificatif.

Concernant la validation du troisième cycle, les trois groupes partagent un noyau dur de convictions : l'épreuve doit être orale devant un jury ; l'entretien doit explorer le cursus de l'interne, analyser son portfolio et examiner la projection du résident à l'égard de sa future pratique professionnelle; les compétences cliniques doivent avoir été évaluées et validées avant la présentation devant le jury, en situation professionnelle ; cette épreuve finale devant jury devra prendre en compte les éléments du "contrôle continu " instauré depuis le début du cursus par les maîtres de stage et le tuteur et qui doivent être rapportés dans le portfolio. Globalement, les analyses des trois groupes convergent pour accréditer l'ancrage du dispositif pédagogique du département de médecine générale au sein du paradigme d'apprentissage et reconnaissent, comme cela a été souligné par ailleurs, qu'il existe "[...] une solide cohérence [...] entre le paradigme d'apprentissage, la perspective constructiviste de l'apprentissage et le courant de l'evaluation authentique "'.

Plusieurs propositions d'amélioration du dispositif de validation formulées par les trois groupes visent à recentrer le rôle du jury autour d'une démarche de jugement global et de décision certificative à porter à l'égard des compétences développées par les étudiants, à partir d'une prise en compte intégrée de multiples sources d'information précédemment rassemblées et interprétées ; il ne devrait pas être de sa responsabilité d'organiser lui-même, à ce moment, des épreuves ou des tests ; ainsi sont suggérées : la suppression de l'épreuve de vignette clinique qui n'a pas lieu d'être si des appréciations récurrentes de la compétence des étudiants ont été préalablement organisées - tant pendant les stages hospitaliers que pendant les stages en médecine ambulatoire - et archivées ; l'évaluation préalable et critériée du portfolio par un enseignant de médecine générale.

Le groupe des responsables institutionnels suggère en outre de réduire la composition du jury à deux membres (un médecin généraliste et un médecin hospitalier), 
dans le but d'améliorer la viabilité organisationnelle du dispositif.

\section{Conclusion}

Dans le cadre d'une redéfinition des orientations pédagogiques du programme de formation du troisième cycle de médecine générale dans notre faculté, nous avons développé un nouveau dispositif expérimental d'évaluation certificative des résidents en fin de cursus. En cohérence avec la perspective constructiviste de l'apprentissage, avec celle de l'enseignement contextualisé et avec celle de l'évaluation authentique, l'usage du portfolio comme outil d'apprentissage, de développement professionnel et d'évaluation a notamment été introduit ; en complément, les résidents ont été invités à présenter et argumenter, lors d'une séance orale terminale devant un jury, une présentation synthétique de leur cursus, des apprentissages réalisés et des compétences développées.

Malgré ses limites méthodologiques, notre travail exploratoire d'évaluation du dispositif expérimental a permis de vérifier : la viabilité opérationnelle du dispositif, son acceptabilité par les résidents et par les différentes catégories de formateurs, sa compréhension par les responsables institutionnels facultaires. Il a également permis de mettre en exergue la nécessité qu'il soit associé à une amélioration de l'accompagnement des résidents dans les nouveaux rôles attendus d'eux : codification et optimisation d'un tutorat périodique, amélioration de la supervision clinique par le développement systématique de procédures récurrentes de feedback et d'évaluation formative des compétences, tant durant les stages hospitaliers que pendant les stages ambulatoires en médecine générale ; formulation de consignes plus explicites pour l'élaboration du portfolio. D'une façon globale, il appa- raît nécessaire de renforcer la cohérence des rôles attendus des différents membres de la communauté d'enseignement, en proposant à tous les formateurs concernés (enseignants du département de médecine générale, maîtres de stages hospitaliers et généralistes) un accompagnement pédagogique visant une meilleure compréhension des orientations pédagogiques retenues et des modalités opérationnelles développées. Enfin, il convient d'améliorer la congruence et la complémentarité de toutes les procédures d'évaluation développées tout au long du cursus et qui concourent à la décision certificative finale.

\section{Remerciements}

Les auteurs remercient les étudiants, les enseignants et les responsables de la faculté de médecine de Nantes, qui ont acceptés de participer à cette expérimentation, de donner un peu de leur temps et de faire partager leurs réflexions.

\section{Contributions}

Pierre Le Mauff a élaboré le protocole de l'évaluation, animé le groupe de discussion des responsables facultaires, analysé le verbatim des trois entretiens et rédigé la première version du manuscrit. Pierre Pottier a participé à l'élaboration du protocole d'évaluation, participé groupe de discussion des enseignants et participé à la rédaction de la version définitive du manuscrit. Lionel Goronflot a participé à la validation du protocole d'évaluation, participé à l'analyse du groupe de discussion des enseignants et à la relecture du manuscrit soumis à la revue. Jacques Barrier a participé à la validation du protocole d'évaluation, animé le groupe de discussion des internes et celui des enseignants, participé au groupe de discussion des responsables de la faculté et a fait une relecture du manuscrit soumis à la revue. 


\section{Recherche et Perspectives}

\section{Annexe 1}

\section{Caractéristiques attendues d'un récit de situation complexe authentique (RSCA)*}

\section{Définition}

C'est un récit, c'est-à-dire que le résident narre une situation qu'il a vécue. Si la forme est libre, le fond doit rendre compte des faits le plus objectivement possible et des perceptions le plus subjectivement possible.

La situation décrite est une situation professionnelle que l'interne a vécue au cours de sa pratique de résident. Elle doit être exemplaire, appartenir au champ de la médecine générale. Elle peut être recueillie en stage hospitalier, en stage de médecine générale, en consultation ou en visite, le résident étant seul ou avec un maittre de stage.

La situation est complexe, c'est-à-dire qu'elle répond aux caractéristiques suivantes :

- la recherche des indices nécessite une exploration minutieuse ;

- elle est multidimensionnelle : biomédicale et/ou psychologique et/ou sociale et/ou éthique et/ou réglementaire et/ou médico-légale ;

- plusieurs stratégies de résolution existent et leur hiérarchisation n’est pas facile ;

- plusieurs solutions peuvent avoir une pertinence comparable ;

- les prises de décisions se font en situation d'incertitude.

Elle est authentique, réellement vécue par l’interne et narrée sans interprétation ni «modifications ».

\section{Modalités}

Le récit est un exercice réflexif du résident à l'égard de sa pratique professionnelle. Elle vise à développer l'auto-évaluation et l'auto-direction de ses apprentissages; dans cette optique, l'étudiant doit pouvoir bénéficier, en tant que de besoin, d'une retroaction (feedback) pédagogique de la part d'un ou plusieurs enseignants. Il est nécessaire de respecter 4 étapes :

- le récit doit contenir les éléments sémiologiques des problèmes perçus, les éléments biographiques et environnementaux du patient, le reflet cognitif et émotionnel chez le résident des informations recueillies, les interactions relationnelles entre le patient, l'interne et l'environnement, les stratégies de résolution de problèmes élaborées par le résident, les éléments du respect de l'autonomie du patient, du consentement éclairé, de la décision partagée, les éléments de la (des) décision(s) prise(s).

- L'analyse de cette situation par le résident "à froid ", sur son action, puis à distance de l'action pour explorer le " comment » et le " pourquoi » des différents actes posés et des différentes décisions prises. Cette phase permet au résident d'identifier ses compétences mais aussi ses limites et ses insuffisances. Il s'agit d'une auto-évaluation de pratique.

- L’auto-formation induite découle de l'auto-évaluation réalisée. Le résident se fixe des objectifs de formation. Il doit décrire cette recherche et doit en préciser les modalités et analyser le niveau de preuve.

- La synthèse reprend les éléments essentiels du travail effectué et doit renseigner sur ce que ce travail réflexif a apporté à l'égard des problèmes à résoudre qui avaient été identifiés.

\section{La rétroaction pédagogique}

C'est une évaluation qualitative et formative du travail réalisé par le résident, à partir d'une grille d'analyse qui prend en compte :

- le récit : l'interne emploie le " je »; le récit reflète le discours de l'autre et relate bien l'action pour permettre à l'enseignant de « chausser les lunettes de l'interne».

- L'auto-évaluation : l'analyse concerne les faits, les perceptions, la relation, la construction des hypothèses, les stratégies de résolution de problème ; le patient est-il intégré à la réflexion et aux décisions ? Le suivi est-il programmé ?

- L'auto-formation : les points à améliorer ont-ils donné lieu à des recherches ? De quelle nature ? Avec quel niveau de preuve? Ces recherches sont-elles transférables à la situation décrite? Le travail est-il pertinent par rapport aux objectifs de formation?

- La synthèse : le travail effectué permet-il d'améliorer la qualité et la pertinence de la résolution des problèmes rencontrés dans la situation décrite?

L'enseignant formule ses commentaires par écrit et, si nécessaire, propose des tâches d'apprentissages complémentaires.

* d'après : Le Mauff P et coll. ${ }^{8}$ 


\section{Annexe 2}

\section{Evaluation des cliniciens enseignants en milieu de soins ambulatoires*}

\section{Nom et prénom du Maître de Stage :}

Période de stage :

Date de l'évaluation :

Chaque intitulé est relatif aux compétences que votre maître de stage (MdS) a mises en œuvre au cours de votre stage de 6 mois.

Entourez un chiffre de 1 à 7 pour chacun des 15 items de la grille ci-dessous.

\begin{tabular}{|c|c|c|c|c|c|c|c|}
\hline Intitulé & $\begin{array}{l}\text { Insatis- } \\
\text { faisant }\end{array}$ & Moyen & $\begin{array}{l}\text { Satis- } \\
\text { faisant }\end{array}$ & Bien & Très bien & Excellent & Supérieur \\
\hline $\begin{array}{l}\text { 1. Qualité des relations du MdS avec } \\
\text { les membres de l'équipe de soins } \\
\text { (secrétaire, associés, correspon- } \\
\text { dants...) }\end{array}$ & 1 & 2 & 3 & 4 & 5 & 6 & 7 \\
\hline $\begin{array}{l}\text { 2. Précision par le MdS de vos atten- } \\
\text { tes et des siennes pour le stage } \\
\text { (définition des responsabilités, } \\
\text { rôles, évaluations etc.) }\end{array}$ & 1 & 2 & 3 & 4 & 5 & 6 & 7 \\
\hline $\begin{array}{l}\text { 3. Bon équilibre entre les soins et } \\
\text { l'enseignement réalisés sur le lieu } \\
\text { de stage }\end{array}$ & 1 & 2 & 3 & 4 & 5 & 6 & 7 \\
\hline $\begin{array}{l}\text { 4. Démonstrations sémiologiques du } \\
\text { MdS }\end{array}$ & 1 & 2 & 3 & 4 & 5 & 6 & 7 \\
\hline $\begin{array}{l}\text { 5. Observation directe par le MdS } \\
\text { de mon aptitude à questionner et } \\
\text { examiner les malades }\end{array}$ & 1 & 2 & 3 & 4 & 5 & 6 & 7 \\
\hline $\begin{array}{l}\text { 6. Observation directe par le MdS } \\
\text { de mon aptitude à expliquer et } \\
\text { enseigner aux malades }\end{array}$ & 1 & 2 & 3 & 4 & 5 & 6 & 7 \\
\hline $\begin{array}{l}\text { 7. Discussion avec le MdS de ma } \\
\text { démarche clinique : analyses des } \\
\text { données et plans de traitement }\end{array}$ & 1 & 2 & 3 & 4 & 5 & 6 & 7 \\
\hline
\end{tabular}




\section{Recherche et Perspectives}

\begin{tabular}{|c|c|c|c|c|c|c|c|}
\hline \multicolumn{8}{|c|}{ Annexe 2 (suite) } \\
\hline Intitulé & $\begin{array}{l}\text { Insatis- } \\
\text { faisant }\end{array}$ & Moyen & $\begin{array}{l}\text { Satis- } \\
\text { faisant }\end{array}$ & Bien & Très Bien & Excellent & Supérieur \\
\hline $\begin{array}{l}\text { 8. Questionnement par le MdS } \\
\text { explorant mon niveau de compré- } \\
\text { hension (Pourquoi ? Si ? Imaginez } \\
\text { que...Puis après ?) }\end{array}$ & 1 & 2 & 3 & 4 & 5 & 6 & 7 \\
\hline $\begin{array}{l}\text { 9. Insistance du MdS sur les « élé- } \\
\text { ments clés " pour chaque malade }\end{array}$ & 1 & 2 & 3 & 4 & 5 & 6 & 7 \\
\hline $\begin{array}{l}\text { 10. Aptitude du MdS à stimuler } \\
\text { mon intérêt et ma curiosité }\end{array}$ & 1 & 2 & 3 & 4 & 5 & 6 & 7 \\
\hline $\begin{array}{l}\text { 11. Identification par le MdS de mes } \\
\text { qualités et de mes forces }\end{array}$ & 1 & 2 & 3 & 4 & 5 & 6 & 7 \\
\hline $\begin{array}{l}\text { 12. Identification par le MdS de mes } \\
\text { besoins d'étude et d'approfon- } \\
\text { dissement }\end{array}$ & 1 & 2 & 3 & 4 & 5 & 6 & 7 \\
\hline $\begin{array}{l}\text { 13. Application par le MdS des } \\
\text { connaissances théoriques aux } \\
\text { malades vus }\end{array}$ & 1 & 2 & 3 & 4 & 5 & 6 & 7 \\
\hline $\begin{array}{l}\text { 14. Démonstration par le MdS de la } \\
\text { prise de décisions diagnostiques } \\
\text { et thérapeutiques « fondées sur } \\
\text { les preuves » }\end{array}$ & 1 & 2 & 3 & 4 & 5 & 6 & 7 \\
\hline $\begin{array}{l}\text { 15. Désir de faire des stages addi- } \\
\text { tionnels avec ce MdS }\end{array}$ & 1 & 2 & 3 & 4 & 5 & 6 & 7 \\
\hline Commentaires libres sur votre stage, & ems c & grille $d$ & luation & autr & ets non & rdés dans & la grille : \\
\hline
\end{tabular}




\section{Annexe 3}

\section{Caractéristiques attendues du portfolio des résidents de médecine générale}

Nous nous sommes appuyés sur des expériences préalablement développées dans d'autres milieux académiques 1,20 comparables au nôtre et sur la définition selon laquelle le portfolio « [...] est une collection de travaux d'un étudiant qui fait foi de ses compétences en gardant des traces pertinentes de ses apprentissages. Les travaux sont sélectionnés conjointement par l'étudiant et l'enseignant $»^{21}$.

\section{Le portfolio}

- Le support : informatique.

\section{- Le contenu :}

- des éléments obligatoires, communs à tous les internes : l’auto-évaluation de début de chaque stage, les objectifs de formation déterminés pour ce stage en interaction avec le(s) maître(s) d'apprentissages (maître de stage/tuteur/enseignants), l'auto-évaluation de fin de stage, deux récits de situation complexe authentique (RSCA) au moins par stage, les comptes-rendus de séances tutoriales institutionnelles.

- des éléments spécifiques à chaque interne : les recherches et travaux personnels, les rétroactions pédagogiques des maîtres d'apprentissages, les notes de synthèse des séances de formation (séminaires, groupes d'échanges de pratique), les tâches demandées par les enseignants, les comptes-rendus de séances tutoriales pédagogiques.

- Les fonctions :

- Outil d'auto-évaluation de la progression du niveau de ses compétences

- Outil de mémoire des internes leur permettant de s’y référer

- Outil d'évaluation formative pour les maîtres d'apprentissages permettant la régulation de leurs interactions pédagogiques

- Outil de supervision transversale, de l'ensemble du curriculum DES (Diplôme d'études spécialisées), pour les tuteurs

- Outil permettant au jury de validation de recueillir un certain nombre de critères d'évaluation

(en vue de la certification).

On comprend ainsi que le portefeuille de compétence est à la fois :

- un outil personnel pour l'interne, sorte de référentiel professionnel en construction,

- un outil pédagogique pour les maîtres d'apprentissage,

- un outil de validation du DES pour le Département de médecine générale et la faculté.

- Propriété : le portfolio appartient à l’interne de médecine générale.

\section{- Niveaux d'utilisation :}

- le niveau d'apprentissage : consultable uniquement par l'interne, c'est son portfolio de travail ou il met tous ses "brouillons",

- le niveau de présentation : consultable par l'interne et son tuteur, c'est le portfolio pédagogique ou les interactions entre l'interne et son tuteur ont lieu (évaluation formative)

- le niveau de validation : consultable par l'interne, son tuteur et les enseignants, c'est le portfolio d'évaluation finale où l'interne et son tuteur présentent à l'institution une sélection de travaux, suivant la réglementation facultaire ${ }^{22}$

\section{Portfolio et validation}

La validation est proposée par un jury multidisciplinaire constitué à parité de représentants des enseignants associés de médecine générale et des maitres de stage (ambulatoires et hospitaliers). Sont pris en compte trois éléments : la validation des stages de la maquette, la soutenance du mémoire de DES, la validation du portfolio de validation présenté par l'interne. Lanalyse du portfolio est faite par un des enseignants associés qui en fait un rapport au jury. 


\section{Recherche et Perspectives}

\section{Références}

1. Jouquan J, Bail P. A quoi s'engage-t-on en basculant du paradigme d'enseignement vers le paradigme d'apprentissage? Pédagogie Médicale 2003;4:163-75.

2. Le MauffP, Urion J, Senand R. Comment les internes de médecine générale sont-ils évalués en fin de DES. La Revue du Praticien - Médecine générale 2006;20:11921.

3. Le Mauff P, Bail P, Gargot F et al. L'évaluation des compétences des internes de médecine générale : aspects théoriques, réflexions pratiques. Exercer 2005;73:63-9.

4. Krueger RA, Casey MA. Focus groups : a practical guide for applied research (3rd. Ed.). London: Sage publications : 2000

5. Moreau A., Dedianne MC, Letrilliart L, Le Goaziou MF, Labarere J, Terra JL. S'approprier la méthode du focus group. La revue du praticien - Médecine générale 2004;645:82-4.

6. Giami A. L'analyse qualitative des entretiens. In: les méthodes qualitatives en psychologie. Paris : Dunod, 2001

7. Pages M., Bonetti M., De Gaulejac V. L'emprise de l'organisation. Paris : PUF, 1999

8. Le Mauff P, Farthouat N, Goronflot L, Urion J, Senand R. Récit de situation complexe et authentique : le modèle nantais. La Revue du Praticien - Médecine Générale 2004;654/655:724-6.

9. Bordage $G$, Zuberi R. Validation d'un instrument d'evaluation des cliniciens enseignants en milieu de soins ambulatoires. $X V^{e}$ Journées internationales francophones de pédagogie médicale. Nancy, 22-25 avril 2003. In : Actes des Journées, 2003 [On-line]. Disponible sur : http://www.cidmef.ubordeaux2.fr/WNancy/com_oral.pdf

10. Lincoln YS, Guba EC. Naturalistic inquiry. Beverly Hills (CA) : Sage, 1985

11. Savoie-Zajc $L$. La recherche qualitativelinterprétative en éducation. In : Karsenti T, Savoie-ZajcL (Coordonnateurs). Introduction à la recherche en éducation. Sherbrooke (QC) : Editions du CRP, 2000: 171-98.
12. Wiggins G. Creating tests worth taking. Educational leadership 1992;49:26-34.

13. Gardner H. Assessment in Context: The Alternative to Standardized Testing. In : Gifford BR and O'Connor MC Changing (Eds.) Assessments : Alternative Views of Aptitude, Achievement and Instruction. Kluwer Academic Publishers, 1992.

14. Jouquan J. Lévaluation des compétences professionnelles des futurs médecins : une exigence sociale et un enjeu pédagogique. Réanimation Urgences 1998;7:611-3.

15. Côté DJ, Bellavance C, Chamberland M, Graillon A. Un programme pour aider les étudiants en médecine à développer leurs stratégies d'apprentissage. Pédagogie Médicale 2004;5:95-102.

16. ABIM, ACP-ASIM et EFIM. Le professionnalisme médical pour le nouveau millénaire: une charte pour les praticiens. Pédagogie médicale 2004;5:43-5.

17. TardifJ. L'évaluation dans le paradigme constructiviste. In: Hivon R. l'évaluation des apprentissages : réflexions, nouvelles tendances et formation. Sherbrooke $(Q C)$ : Editions du CRP 1993:27-56.

18. Naccache N, Samson L, Jouquan J. Le portfolio en éducation des sciences de la santé : un outil d'apprentissage, de développement professionnel et d'évaluation. Pédagogie Médicale 2006;7:110-27.

19. Jouquan J. L'évaluation des apprentissages des étudiants en formation médicale initiale. Pédagogie Médicale 2002;3:38-52.

20. Bail P, Le Reste JY, Boiteux F. Le Portfolio, expérience du Département de médecine générale de la Faculté de Brest La Revue du Praticien - Médecine générale 2004;646:445-7.

21. Ministère de l'éducation du Québec "portfolio sur support numérique » document d'information; mai 2002. htpp ://www.meq.gouv.qc.ca.I

22. Le D.E.S. de médecine générale nantais, 2004. [On-line]. Disponible sur : www.sante.univ nantes.fr/med/medgen

Manuscrit reçu le 9 mars 2005 ; commentaires éditoriaux formulés aux auteurs le 12 mai 2005 et le 29 juin 2006 ; accepté pour publication le 5 juillet 2006 